\section{Rectis}

Revista Colombiana de Ciencia Animal ISSN: 2027-4297

reciaeditor@unisucre.edu

Universidad de Sucre

Colombia

\title{
Manejo de suelos en el acuífero Morroa, Sucre, Colombia, alternativa para el desarrollo agrícola sostenible
}

\author{
Vergara F, Vicente \\ Manejo de suelos en el acuífero Morroa, Sucre, Colombia, alternativa para el desarrollo agrícola sostenible \\ Revista Colombiana de Ciencia Animal, vol. 11, núm. 1, 2019 \\ Universidad de Sucre, Colombia \\ DOI: https://doi.org/10.24188/recia.v0.n0.2019.711 \\ Los autores permiten a RECIA reimprimir el material publicado en él. En caso de que un autor quiera traducir o \\ usar una publicación parcial o completa de nuestro Diario, el autor debe obtener un permiso por escrito del editor \\ de la revista. \\ Esta obra está bajo una Licencia Creative Commons Atribución 4.0 Internacional.
}




\section{Manejo de suelos en el acuífero Morroa, Sucre, Colombia, alternativa para el desarrollo agrícola sostenible}

Soil management in the Morroa aquifer, Sucre, Colombia, alternative for sustainable agricultural development

Vicente Vergara F

DOI: https://doi.org/10.24188/recia.v0.n0.2019.711

Universidad de Sucre. Grupo de investigación Conservación

del Recurso Hidrico y de Alimentos "CRHIA, Colombia

viceunisucre@yahoo.com

iD http://orcid.org/0000-0002-6752-6555

Recepción: 29 Diciembre 2018

Aprobación: 28 Febrero 2019

Publicación: 06 Marzo 2019

\section{RESUMEN:}

En un predio ubicado en el municipio de Morroa, departamento de Sucre, en el Caribe colombiano, fueron construidas siete tipos de obras de bioingeniería, con el fin de rehabilitar los suelos y favorecer potencialmente la recarga del acuífero Morroa por aumento de la infiltración. El presente estudio describe las características de las obras construidas y la eficiencia de cada una en el cumplimiento de su fundamento técnico. Asimismo, fue evaluado el porcentaje de adaptación del material vegetal empleado en dichas obras. En términos generales los resultados técnicos obtenidos fueron satisfactorios, aunque es fundamental realizar educación ambiental y fortalecer el ejercicio de la autoridad con la comunidad de la zona, para garantizar la sostenibilidad de las obras de bioingeniería construidas.

Palabras Clave: Aguas subterráneas, bioingeniería, erosión, obras, rehabilitación.

\section{ABSTRACT:}

In a property located in the municipality of Morroa, department of Sucre, in the Colombian Caribbean, seven types of bioengineering works were built, in order to rehabilitate the soils and potentially favor the recharge of the Morroa aquifer by increasing infiltration. The present is study that describes the characteristics of the constructed works and the efficiency of each in the fulfillment of its technical foundation. Likewise, the percentage of adaptation of the plant material used in said works was evaluated. In general terms, the technical results obtained were satisfactory, although it is essential to carry out environmental education and strengthen the exercise of authority with the community of the area, in order to guarantee the sustainability of the constructed bioengineering works.

KEYWORDS: Groundwater, bioengineering, erosion, works, rehabilitation.

\section{INTRODUCCIÓN}

La erosión del suelo es la pérdida de su capa superficial por el arranque, transporte y deposito del mismo. Ésta es generada básicamente por el impacto de la lluvia y las aguas de escorrentía o por efecto del viento. Asociados a la erosión del suelo se encuentran los conceptos de erodabilidad y erosividad. La erosividad es la capacidad que tiene la lluvia para generar erosión, en función de su intensidad e independientemente al tipo de suelo. Por otra parte, la erodabilidad es la susceptibilidad que tiene un suelo a erosionarse, ésta depende de sus condiciones físicas $(K)$, longitud de pendiente $(L)$, gradiente de pendiente $(S)$, factor de cobertura vegetal $(C)$ y prácticas de manejo realizadas sobre él $(P)(1)$. Siendo estos dos parámetros finalmente mencionados los que puede controlar el hombre en las cuencas hidrográficas y en particular en las zonas de recarga de acuíferos para contribuir al desarrollo sostenible en los mismos $(2,3)$.

En el marco de lo anterior las malas prácticas agropecuarias aumentan la erodabilidad del suelo, debido a que en primera medida remplazan o retiran la cobertura vegetal. Posteriormente la ganadería y la agricultura, 
durante su desarrollo afectan el comportamiento del agua de las precipitaciones sobre el suelo, aumentando la escorrentía y disminuyendo la infiltración $(4,5)$.

El acuífero Morroa está ubicado en el norte de Colombia y es la fuente más importante de abastecimiento de agua y desarrollo para las comunidades de los municipios que lo circunscriben; como Sampués, Sincelejo, Corozal, Morroa, Los Palmitos y Ovejas, en el departamento de Sucre; Sahagún y Chinú, en Córdoba; y El Carmen de Bolívar, en el departamento Bolívar. En el área de recarga de ese cuerpo de agua la cobertura vegetal y las prácticas de manejo y conservación de suelos son afectadas por las malas prácticas agrícolas, este hecho compromete su sostenibilidad (6).

Asimismo, Vergara et al (6) identificaron que el 7,4\% del área del acuífero Morroa presenta una vulnerabilidad alta a contaminación por plaguicidas. Por lo anterior, Vergara (7) recomendó implementar alternativas de manejo ambiental en dichas áreas vulnerables correspondientes a zonas rurales de los municipios involucrados.

Tipos de erosión de suelos.

Erosión laminar. Es el arrastre de suelo en capas superficiales bien definidas generado por acción de la escorrentía. Este tipo de erosión es muy común en las zonas recientemente deforestadas y en los suelos residuales; y es dado de manera casi imperceptible. El proceso inicia con el impacto de las gotas de lluvia que altera el suelo superficial. Luego parcialmente el agua infiltra y es acumulada sobre la superficie del terreno, formándose una corriente de agua de 2 a 3 milímetros de espesor. El flujo laminar es poco profundo en las partes más altas de la ladera pero la profundidad aumenta en las zonas más bajas. La corriente propiamente laminar tiene poco poder erosivo pero al continuar la lluvia y al mismo tiempo ocurrir el flujo genera turbulencia, aumentando la capacidad de erosión.

Son extraordinariamente susceptibles a la erosión laminar las áreas de cultivos no permanentes, al igual que los suelos sin vegetación y los que están sujetos a sobrepastoreo $(1,8)$.

Erosión por surcos. Es una forma de erosión fácilmente perceptible, ocurre cuando la escorrentía empieza a concentrarse sobre la superficie del terreno, debido a la irregularidad natural que puede poseer. Inicialmente son formados pequeños surcos o canales prácticamente imperceptibles en forma de $\mathrm{V}$. Con el paso del tiempo se van volviendo más profundos y la energía del agua en movimiento adquiere cada vez más fuerza. Al desprenderse y transportarse más partículas de suelo dentro del surco, éste puede tomar forma de $U(1,9)$.

Erosión por cárcavas. Es denominada cárcava a un canal de erosión con una sección superior a un pie ${ }^{2}$. Son formadas al profundizarse y ampliarse los surcos, de hecho es el estado más avanzado de ese tipo de erosión. Asimismo, varios pequeños surcos pueden unirse y crecer para formar una cárcava (1).

Las cárcavas actúan como cauces de concentración y transporte de agua y sedimentos, que generalmente llevan agua sólo durante e inmediatamente después de lluvias de gran intensidad (10).

Las áreas más susceptibles a erosión en cárcavas son aquellas de alta pendiente y horizontes de suelo de gran espesor. Las mesetas semiplanas que recogen gran cantidad de aguas de escorrentía y a su vez tienen taludes de pendiente lateral fuerte presentan especial susceptibilidad a la formación de cárcavas, especialmente anchas. Asimismo, las áreas más afectadas por carcavamientos son aquellas que tienen suelos dispersivos o altamente erodables. Por otra parte, la formación de cárcavas frecuentemente aparece ligada a incrementos en las escorrentías. Dicho incremento, puede deberse a cambios en el uso del suelo, o modificaciones en el área de drenaje motivadas por el hombre, a eventos de precipitación de características extremas, entre otras causas $(1,11)$.

Remoción masal. Son los fenómenos de desprendimiento, transporte y depositación de grandes masas de suelo por acción de la fuerza del agua en movimiento. En su orden sería el tipo de erosión más perceptible y con peores consecuencias antrópicas, pues además de empobrecerse los suelos, aguas abajo de ellos, pueden ser nefastas las secuelas sobre el hombre, sus bienes y demás elementos de desarrollo. 
En el desprendimiento y transporte de las masas actúan las fuerzas de gravedad y la fuerza del agua procedente de las lluvias. Por parte del suelo influyen el gradiente topográfico, la sismicidad y la meteorización de la roca parental $(1,12)$.

Terracetas por sobrepastoreo. Las terracetas por sobrepastoreo son el tipo de erosión cuya única causa es la ganadería principalmente extensiva, son generadas por la compactación del suelo, que a su vez es producida por las pisadas constantes de los animales. Cuanto más pendiente tengan los suelos son mayormente susceptibles a ese tipo de erosión. Este fenómeno erosivo es quizás el único donde las principales causantes no son las lluvias de gran intensidad. Aunque a partir de las terracetas por sobrepastoreo se puede generar remoción masal por acción de las lluvias $(4,5)$

Erosión del suelo y la recarga de los acuíferos. La erosión del suelo es consecuencia de la desaparición o disminución de la cobertura vegetal. El proceso inicia cuando la infiltración es superada por la escorrentía. Los caudales de esta última se acrecientan con el paso del tiempo y por ende la remoción y el transporte de las partículas físicas y químicas que componen el suelo. Consecuentemente disminuyen los caudales o láminas de infiltración, lo cual terminaría afectando negativamente la recarga de los acuíferos. Por otra parte, al erosionarse los suelos pierden su capacidad productiva lo que dificulta cada vez más la posibilidad de que exista vegetación, hecho que contribuye también a la disminución de la infiltración, percolación y recarga de las aguas subterráneas $(3,13)$.

Con la intención de mitigar o prevenir la erosión, rehabilitar los suelos y por ende favorecer la infiltración en las zonas de recarga del acuífero Morroa, fueron construidas siete tipos obras de bioingeniería. En el presente artículo tiene por objetivo presentar los mecanismos de construcción de las obras de bioingeniería, la selección técnica de cada una de acuerdo al fenómeno erosivo a manejar, la evaluación de adaptación del material vegetal y la sostenibilidad de las mencionadas obras.

\section{MATERIALES Y MÉTODOS}

Área de estudio. El presente corresponde a un estudio de caso realizado en la zona rural del municipio de Morroa, en un predio de propiedad del Municipio de Sincelejo donde éste construyó una obra recarga de aguas subterráneas. Morroa está ubicado al norte de Colombia, en la Costa Caribe geográficamente en las coordenadas $9^{\circ} 20^{\prime} 01^{\prime \prime} \mathrm{N} 75^{\circ} 18^{\prime} 24^{\prime \prime} \mathrm{W}$, presenta paisaje variable entre montaña y lomerío, con una altura sobre el nivel del mar de $160 \mathrm{~m}$, edafológicamente está constituido por areniscas calcáreas y calizas, la temperatura promedio es de $27^{\circ} \mathrm{C}$, presenta precipitaciones entre los 1000 y los $1300 \mathrm{~mm}$ y una humedad media anual de $70 \%$ (14).

Identificación de los tipos de erosión para definir la obra de bioingeniería a construir. El estudio fue realizado durante un año, iniciando con recorridos técnicos en el sitio con el fin de identificar los tipos de erosión existentes y definir la obra de bioingeniería a construir para el manejo del fenómeno erosivo observado y la rehabilitación de los suelos afectados por el mismo.

Construcción de las obras de bioingeniería. Las obras de bioingeniería construidas fueron cajas de disipación, toboganes vegetativos, acequias de contorno, trinchos de contención, presas de varas, cajuelas de humificación y biomantos artesanales. El material vegetativo utilizado fue matarratón (Gliricidia sepium) mayoritariamente, jobo (Spondias mombin) y uvito (Cordia alba) en menores a cantidades; teniendo en cuenta su reproducción asexual.

Según Cardozo (15), Gliricidia sepium es una leguminosa arbórea y perenne, por lo tanto es caracterizada por fijar nitrógeno atmosférico en sus nódulos radicales para luego almacenarlos por medio de su metabolismo a su componente forrajero. Puede llegar alcanzar una altura de 12 metros con un diámetro basal que oscila de 40 a 70 centímetros de diámetro. Es nativa desde México hasta la parte norte de América del Sur, encontrándose ampliamente distribuido en las regiones tropicales del mundo. Es común encontrarlo en climas cálidos y medios, crece desde el nivel del mar hasta los $1500 \mathrm{~m}$ de altitud, con precipitaciones de 600 
a $8000 \mathrm{~mm} /$ año, en suelos ácidos, salinos, arenosos y hasta infértiles. Sin embargo, no tolera niveles freáticos altos o con mal drenaje interno. Tolera una gama amplia de suelos, desde arenas puras hasta vertisoles negros profundos, con un $\mathrm{pH}$ de 4 a 7 . Presenta un desarrollo adecuado a temperaturas entre 20,7 y $29,2^{\circ} \mathrm{C}$, pero probablemente reduzca su crecimiento y se defolie si estas son inferiores a $15^{\circ} \mathrm{C}$. Es usada como alimento para animales, constituyéndose en una alternativa interesante de fuente proteica en la alimentación de rumiantes.

Cajas de disipación. Éstas fueron construidas mediante excavaciones de $1 \mathrm{~m}^{2}$ por $70 \mathrm{~cm}$ de profundidad y revestidas en sus lados por listones de material vegetativo ubicados de forma horizontal, los cuales fueron fijados por otros entrados verticalmente en el suelo. Las cajas de disipación fueron ubicadas según trazado y replanteo perpendicular al flujo del agua superficial en el terreno.

Toboganes vegetativos. Estas obras son un revestimiento sobre los surcos del suelo mediante material vegetativo ubicado de forma horizontal y vertical, los cuales son atados mediante alambre quemado para garantizar la estabilidad mientas se da el proceso de retoño. Para su construcción es necesario perfilar el surco de tal manera que tome forma regular y el revestimiento de estructura sea total.

Acequias de contorno. Las acequias de contorno fueron construidas mediante excavaciones de 30 centímetros de ancho por 30 centímetros de profundidad, realizadas en el sentido de las curvas de nivel del suelo. Al momento de hacer la excavación fue indispensable disponer los residuos aguas abajo de cada estructura.

Trinchos de contención. Son construidos mediante listones de material vegetativo de reproducción asexual, que para el caso remplazarían al acero y concreto usados normalmente en los tradicionales muros de contención. Son el resultado de incrustar de forma vertical cada $25 \mathrm{~cm}$ listones de un metro de longitud, en una excavación de $50 \mathrm{~cm}$ de profundidad y $25 \mathrm{~cm}$ ancho aproximadamente. Dicha excavaciones fueron ubicadas, en las zonas susceptibles a remoción masal, perpendicularmente a los flujos de las escorrentías y rellenadas con estacas del mismo material ubicadas en forma horizontal hasta sobresalir aproximadamente $30 \mathrm{~cm}$ del nivel del suelo.

Presas de varas. fueron construidas mediante listones de material vegetativo de $0,3-1$ metro de longitud, incrustados de forma vertical, cada $25 \mathrm{~cm}$ aproximadamente. Luego a estos mediante alambre fueron atadas perpendicularmente otras estacas de longitud según el ancho de la corriente o surco de gran tamaño a manejar. Estos listones quedaron pegados unos con otros para que pudieran disminuir la velocidad del agua que fluiría en la corriente. Aguas abajo de esta estructura fueron colocadas sobre la superficie del suelo una serie de estacas acostadas, atadas entre sí mediante alambre y fijadas a través de otras de menor longitud $(30 \mathrm{~cm})$ enterradas a distancias de $25 \mathrm{~cm}$ aproximadamente. Este último arreglo se denomina "delantal", dentro de la estructura. Fueron construidas en aquellos sitios de corrientes de gran tamaño, ubicadas según trazado y replanteo perpendicular al flujo del agua superficial en el terreno.

Cajuelas de humificación. fueron construidas mediante excavaciones sobre el terreno de 30 centímetros de ancho por 30 centímetros de profundidad. Las cajuelas de humificación fueron rellenadas con materia orgánica procedentes de residuos de cosecha, de limpieza o desmalezado de lotes, de limpieza de potreros y de otras actividades del campo como por ejemplo la sacada de punta de material vegetal utilizado en las obras de bioingeniería.

Biomantos artesanales. Para su implementación fueron identificadas las porciones del terreno afectadas por la erosión laminar, en esas áreas fueron colocados sacos de fique sobre la superficie fijados mediante estacas de material vegetativo de forma vertical.

Seguimiento a la adaptación del material vegetal y a las mismas obras de bioingeniería. Posterior a la construcción de las obras de bioingeniería fue realizado seguimiento al retoño y adaptación del material vegetal utilizado, asimismo fue evaluada la eficiencia en el cumplimiento del objetivo para el que fue construida cada una. 


\section{RESULTADOS}

En el área de estudio fueron determinados fenómenos erosivos de diferentes tipos. Fue identificada erosión por surcos, en algunos sitios en su etapa inicial y en otros en etapas más avanzadas, lo que in situ es identificado por el ancho del surco que en algunos casos es inferior a un metro y en otros alcanza hasta los 10 metros.

Asimismo, fue identificada erosión laminar, principalmente en las cotas más altas, carcavamientos y remoción masal mayoritariamente en las partes bajas y medias del terreno.

Lo anterior dio origen a la construcción de obras de bioingeniería según la necesidad de manejo y rehabilitación presentada por áreas de suelos.

Cajas de disipación. Estas estructuras controlan la erosión por surcos, cuando el ancho del canal supera los 10 metros, de la misma forma pueden manejar la erosión laminar. Las cajas de disipación funcionan interceptando la escorrentía, disminuyendo su energía cinética, reteniendo el agua, aumentando la infiltración y reduciendo el caudal que escurre por la superficie del suelo (Figuras 1, 2 y 3 ).

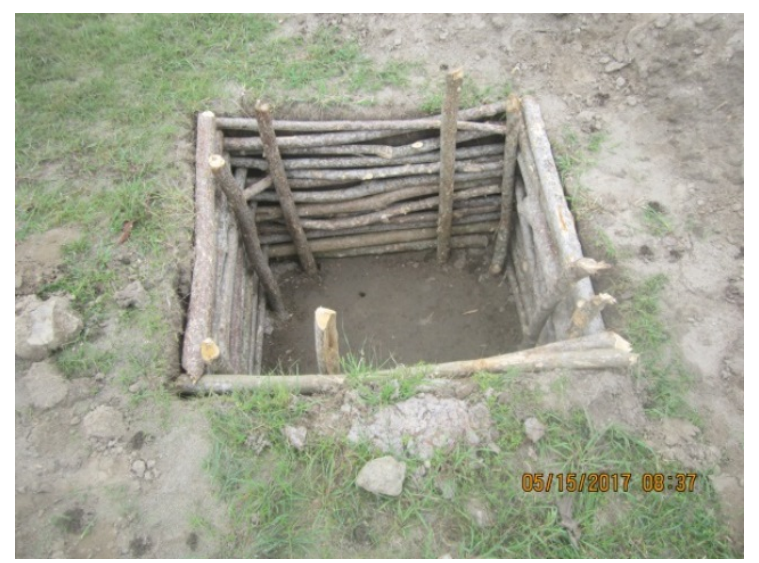

FIGURA 1.

Cajas de disipación construidas.

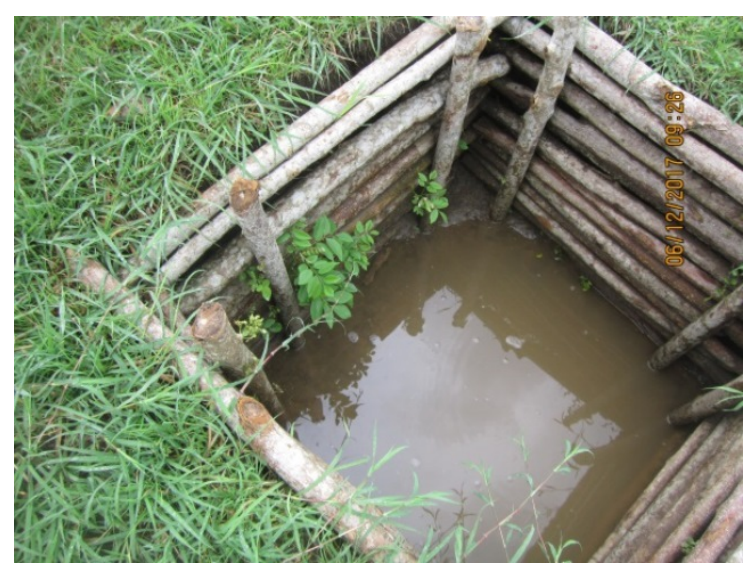

FIGURA 2.

Inicio del retoño de material vegetal en cajas de disipación y retención de agua lluvia. 


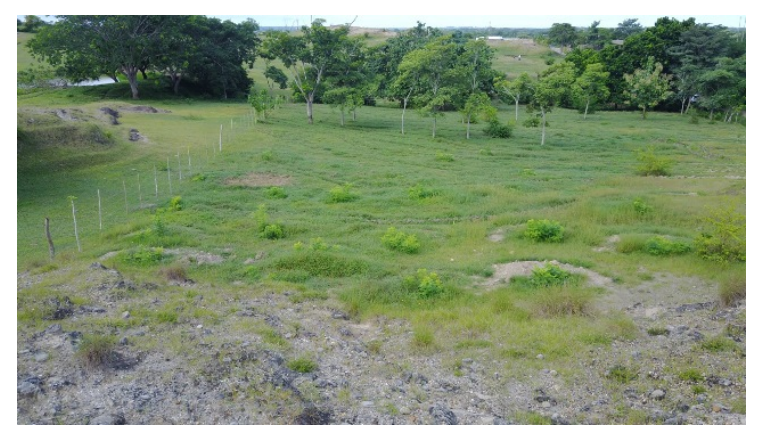

FIGURA 3.

Cajas de disipación con material vegetal retoñado.

El resultado de las cajas de disipación construidas fue satisfactorio, por un lado el material vegetal retoñó en un $80 \%$ en menos de dos meses y durante toda la temporada de lluvias las aguas de la escorrentía fueron retenidas en dichas estructuras sin sobrepasar la capacidad de cada una.

Toboganes vegetativos. Los toboganes vegetativos son obras de bioingeniería cuyo fundamento técnico inicialmente es impedir el contacto directo entre las escorrentías y el suelo, para de esta forma manejar la erosión por surcos. En el estudio fueron construidos en los surcos que no superaron un metro de ancho (Figura 4).

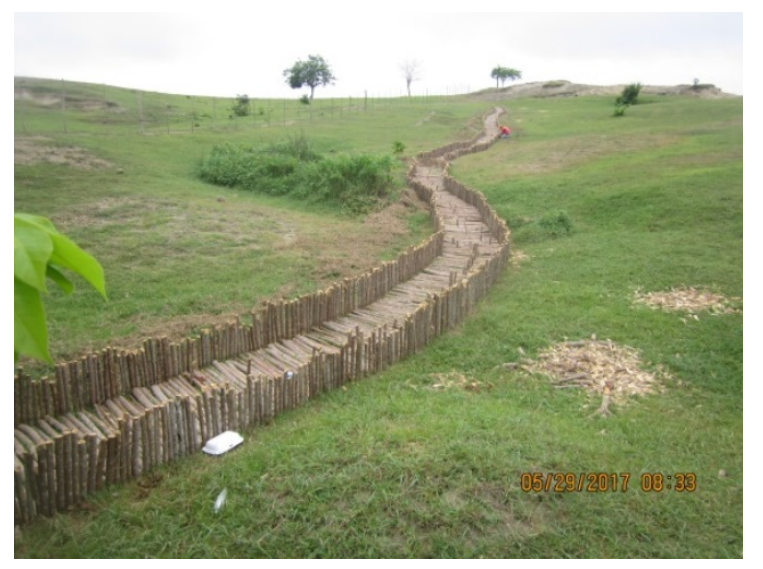

FIGURA 4.

Tobogán vegetativo construido.

Con el retoño y posterior crecimiento del material vegetal que conforma los toboganes vegetativos, dichas estructuras además de evitar el contacto directo entre el suelo y las aguas de escorrentía, aumentan la infiltración (Figura 5). 


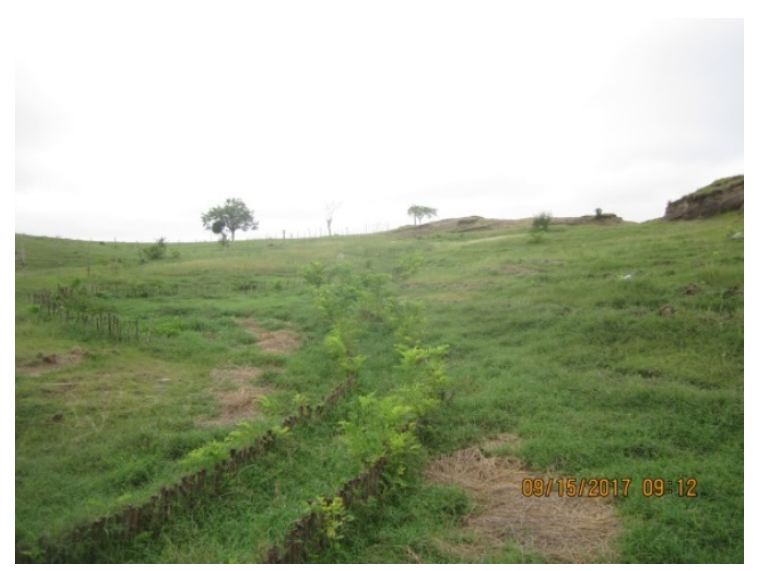

FIGURA 5.

Tobogán vegetativo, material vegetal retoñado.

El resultado de los toboganes vegetativos construidos fue excelente, puesto que a los cuatro meses en el 100\% del área cubierta con esas estructuras retoñó el material vegetal utilizado (Figura 6).

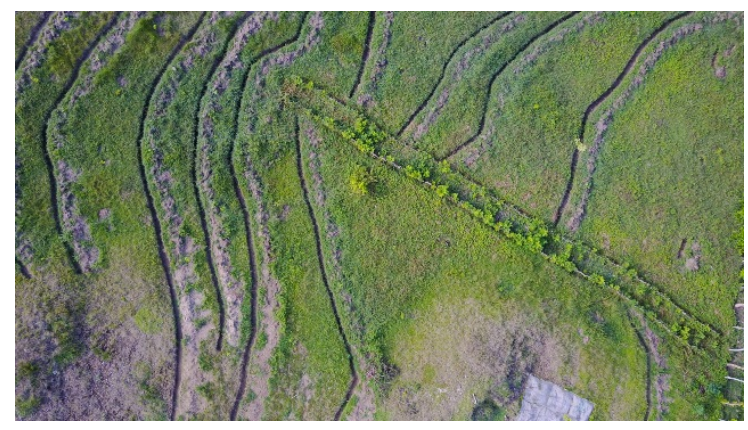

FIGURA 6.

Tobogán vegetativo con material vegetal retoñado.

Acequias de contorno. Estas estructuras controlan la erosión laminar. $\mathrm{Al}$ igual que las cajas de disipación funcionan interceptando la escorrentía, disminuyendo su energía cinética, reteniendo el agua, favoreciendo la infiltración, disminuyendo el caudal que escurre por la superficie del suelo y manteniendo la humedad del suelo. En el estudio las acequias de contorno fueron conectadas con a los toboganes vegetativos, obteniéndose excelentes resultados (Figuras 7,8 y 9 ).

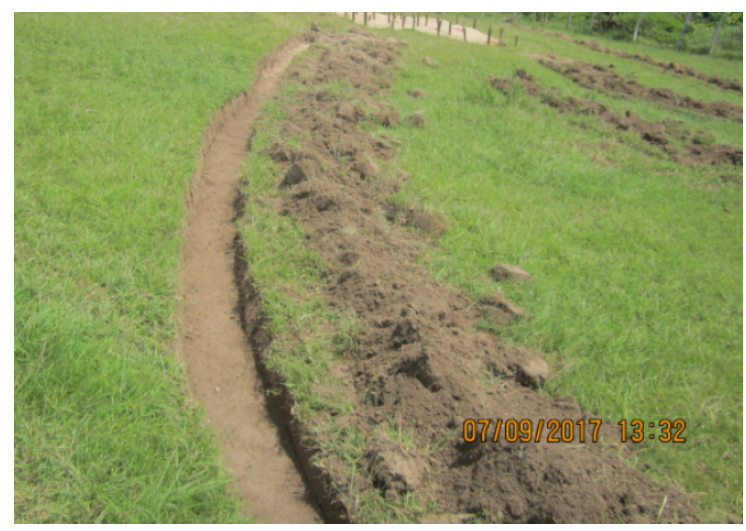

FIGURA 7.

Acequias de contorno construídas 


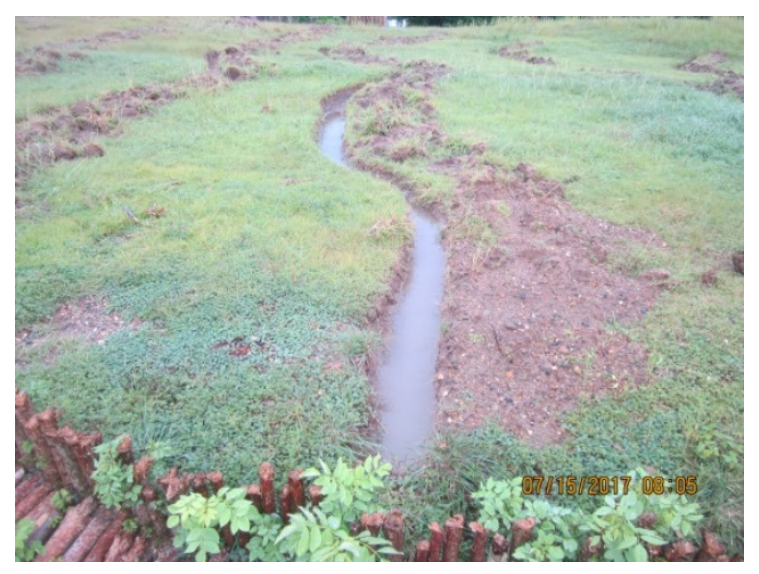

FIGURA 8.

Eficiencia de acequias de contorno ante las precipitaciones.

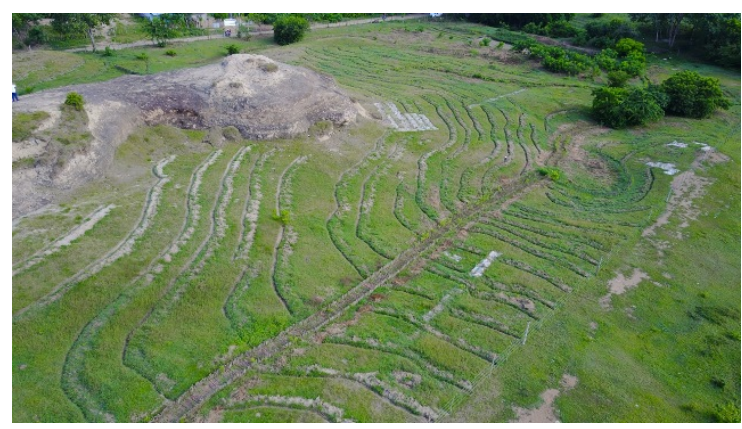

FIGURA 9.

Acequias de contorno conectadas con toboganes vegetativos.

Las acequias de contorno arrojaron una alta eficiencia. En primera medida no hubo escorrentía al interior de cada una, eso indica un excelente trabajo de trazado y excavación. Asimismo, todas retuvieron el agua de las escorrentías sin sobrepasar la capacidad de cada una durante toda la temporada de lluvias.

Trinchos de contención. Las anteriormente mencionadas malas prácticas agropecuarias generan sobre los suelos varias consecuencias negativas enmarcadas dentro lo denominado erosión. Dicho fenómeno de impacto ambiental tiene varias causas y consecuencias, así por ejemplo los suelos de laderas donde fueron realizadas prácticas de ganadería extensiva son afectados por un fenómeno erosivo, ya citado, denominado terracetas por sobrepastoreo, que más adelante las precipitaciones potencializan a través de la infiltración en su origen, generando un impacto mayúsculo llamado remoción en masa o remoción masal. En el sitio del proyecto como medida de manejo para ese tipo de erosión y del carcavamiento fueron construidos los trinchos de contención, las cuales son auténticas obras de ingeniería más resistente con el paso del tiempo porque crece el material vegetal que las compone (Figuras. 10 y 11). 


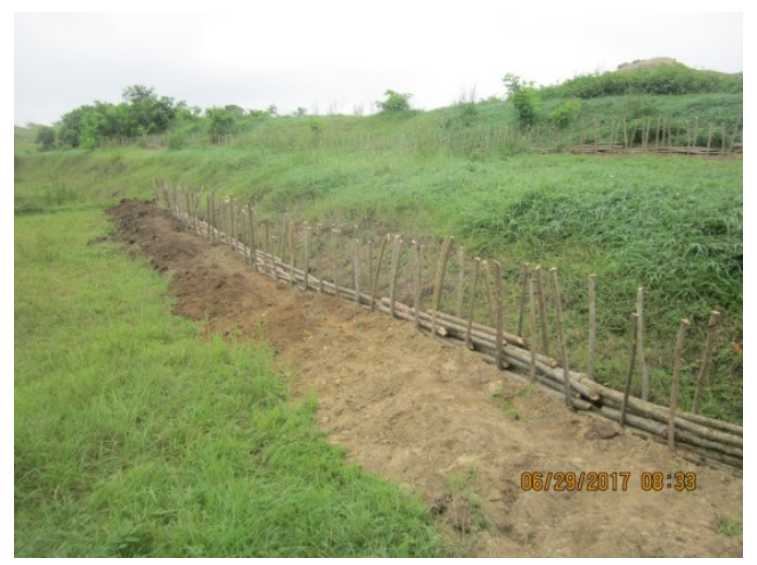

FIGURA 10.

Trinchos de contención construídos.

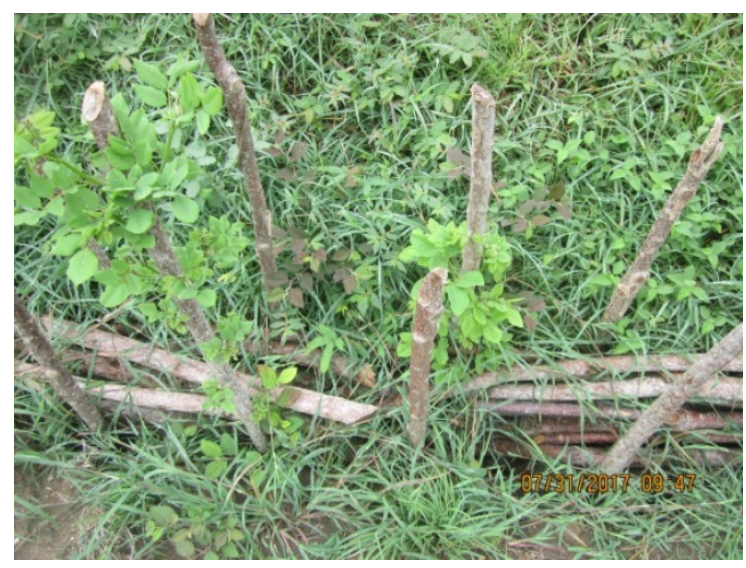

FIGURA 11.

Retoño de material vegetal en trinchos de contención.

Presas de varas. Resaltando que la erosión por surcos es generada en sentido perpendicular a las curvas de nivel del terreno en los suelos de laderas debido a las precipitaciones de gran intensidad. Con el paso del tiempo las precipitaciones agrandan los mencionados surcos convirtiéndolos casi en arroyos transitorios. Ese tipo de erosión fue manejado en el proyecto mediante presas de varas. Estas obras de bioingeniería tienen como principio técnico reducir la energía cinética de las escorrentías en lugares donde fluyen grandes corrientes de agua y de esa forma mitigar el fenómeno erosivo que acrecentaría el tamaño los mencionados arroyos (Figura 12). 


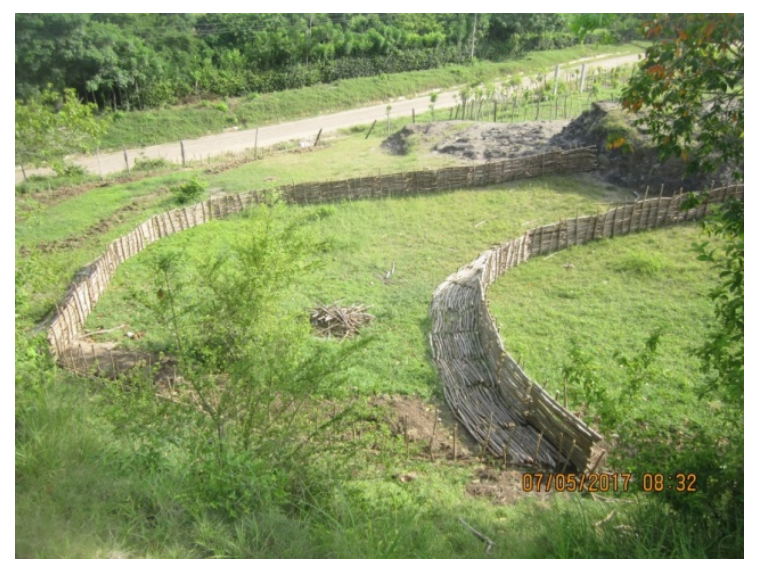

FIGURA 12.

Presas de varas construídas.

El resultado de las presas de varas construidas fue satisfactorio, a pesar de que el material vegetal retoñó solo en un 40\%, este proceso inició antes del primer mes. Es importante resaltar que por el mismo sistema de construcción quedan algunos listones horizontales sin contacto con el suelo. El éxito de la estructura está en que los listones verticales retoñaron todos y los horizontales que están en contacto con el suelo retoñaron mayoritariamente. Al pasar el tiempo los retoños serán árboles que cumplirán la función de la estructura construida inicialmente (Figuras 13 y 14 ).

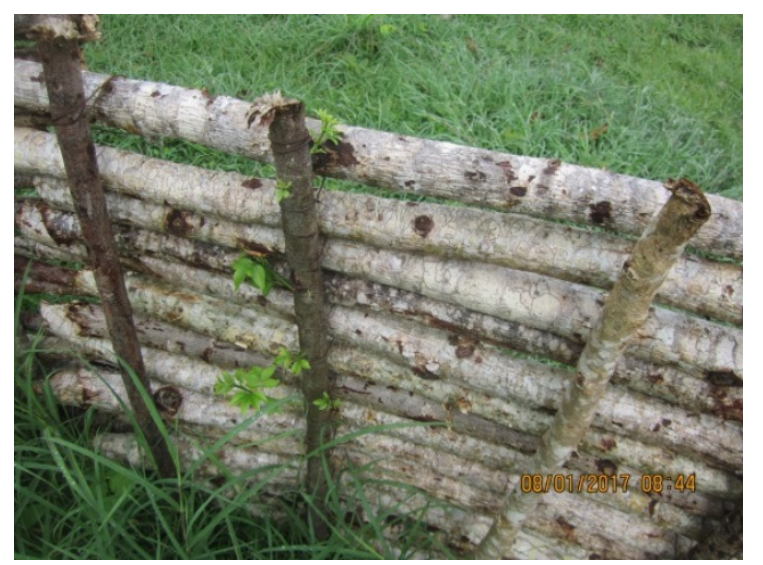

FIGURA 13.

Rebrote de material vegetal vertical en presas de varas construídas. 


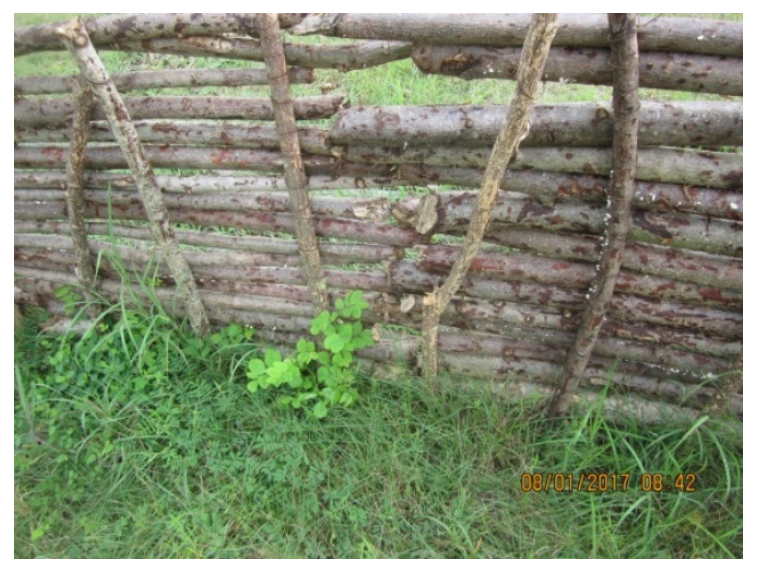

FIGURA 14.

Rebrote de material vegetal horizontal en presas de varas construidas.

Cajuelas de humificación. En el sitio del estudio fueron construidas cajuelas de humificación teniendo en cuenta que los suelos, junto a fenómenos erosivos físicos, padecen también de pérdida de nutrientes. El objetivo principal de esas estructuras es favorecer la formación de humus en el suelo y de esa forma rehabilitar su capacidad productiva a nivel ecosistémico (Figuras 15 y 16).

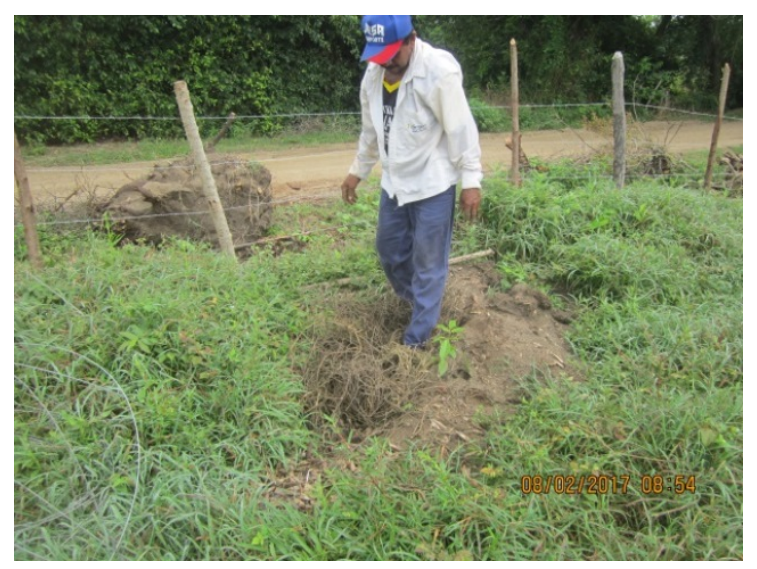

FIGURA 15.

Cajuelas de humificación rellena con los residuos del material vegetal de limpieza de lotes. 


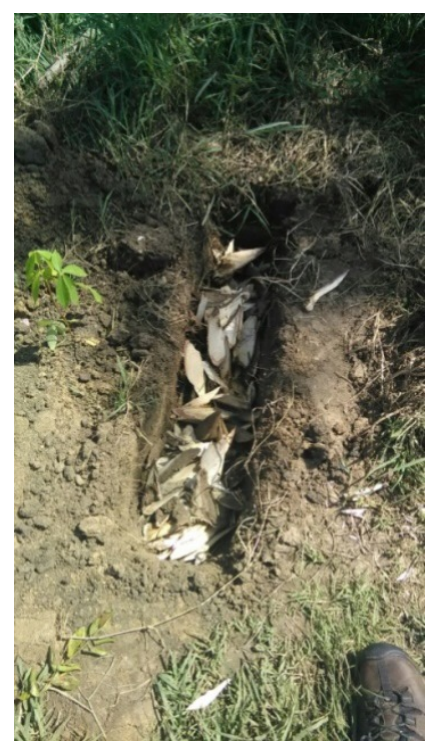

FIGURA 16.

Cajuelas de humificación con los residuos del material vegetal de la construcción de obras de bioingeniería.

En cuanto a la eficiencia de las cajuelas de humificación, el material orgánico mostró una rápida descomposición debido a las ya descritas condiciones climáticas del área de estudio, que favorecen la actividad de los microorganismos. Esto indica una rápida humificación de la materia orgánica, lo que en el suelo mejora su estructura, la penetración y la retención del agua; favorece el intercambio gaseoso, disminuye la erodabilidad, aumenta la capacidad de intercambio catiónico y la capacidad tampón. Eso redunda en un aumento de su fertilidad global (16).

Biomantos artesanales. Los biomantos artesanales son una forma de prevenir, mitigar y remediar la erosión laminar en los suelos. Con esa medida es evitado el contacto directo entre el agua de las precipitaciones y el suelo, mediante los sacos de fique que lo protegen mientras el material vegetal es desarrollado (Figuras. 17 y 18$)$.

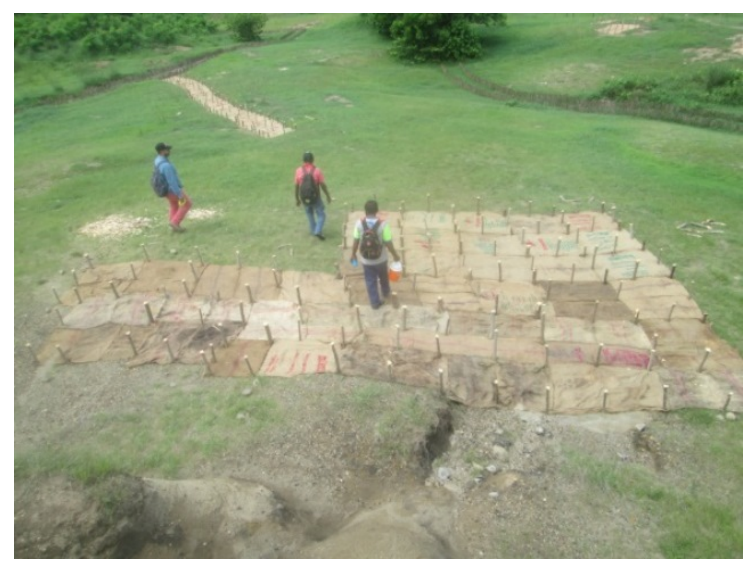

FIGURA 17.

Biomantos artesanales construidos. 


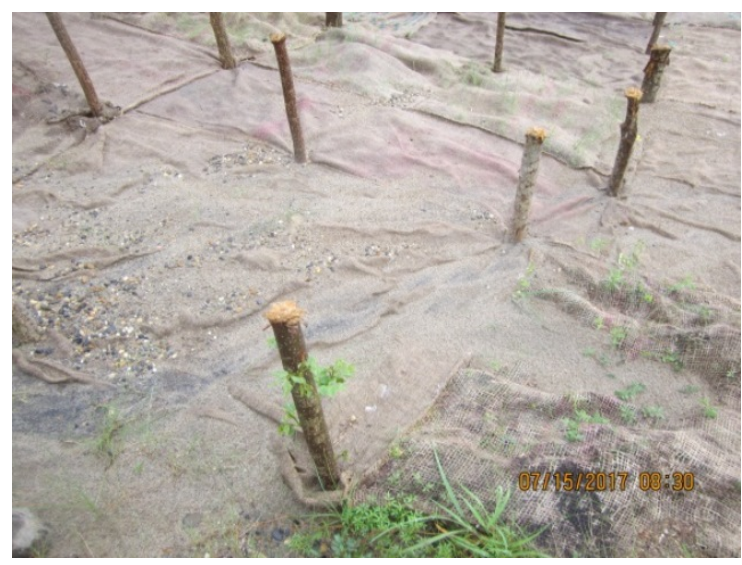

FIGURA 18.

Rebrote de material vegetal en biomantos artesanales.

El resultado de las biomantos artesanales construidos en el sitio de estudio fue satisfactorio, por un lado el material vegetal retoñó desde el primer mes obteniéndose una eficiencia final del 75\% y la humedad retenida en los sacos favoreció la aparición de arvenses en áreas donde antes no era posible.

\section{DISCUSIÓN}

Las obras de bioingeniería son estructuras sostenibles, debido a que el paso del tiempo las hace más fuertes. Además de favorecer la rehabilitación de los suelos contribuyen a la recarga de las aguas subterráneas, convirtiéndose en aporte a la sostenibilidad del acuífero Morroa. En complemento de lo anterior Flórez (17) manifiesta que las obras de bioingeniería son ambientalmente amigables y embellecen el paisaje, ya que devuelven el verde natural al sitio tratado y a su área de influencia, mejorando la diversidad genética de la región.

De la Ossa et al (18) describen un aumento de la demanda de alimentos a nivel mundial, hecho que sometería a los elementos ambientales, principalmente al suelo, a una mayor presión. Por tal razón es indispensable el uso sostenible de dicho recurso y del agua durante la producción agropecuaria, que podría alcanzarse mediante el desarrollo de tecnologías limpias que reduzcan el uso del agua y generen proceso de rehabilitación y conservación del suelo agrícola. De acuerdo a esto, son las obras de bioingeniería excelentes alternativas tecnológicas de apoyo a las prácticas ganaderas y agrícolas para la sostenibilidad del suelo.

En las zonas pertenecientes al trópico ecuatorial las obras de bioingeniería, principalmente las que poseen material vegetal, deben ser construidas al iniciar la primera temporada de lluvias porque requieren de la presencia del agua para su retoño, enraizamiento y adaptación en general. Por otra parte, las obras de bioingeniería compuestas por la especie leguminosa Gliricidia sepium, además de todo los beneficios físicos descritos, fijan nitrógeno en el suelo siendo esto un aporte positivo a su fertilidad (19).

Con la construcción de las obras de bioingeniería, en el periodo mencionado como ideal para ello, en poco tiempo la apariencia de los lotes mejora en cuanto a la presencia de vegetación. Dicho aspecto perdura aun en la época de sequía aunque la rehabilitación de los suelos apenas inicie. En ese sentido, mientras en los otros predios agropecuarios de la zona determinada predomina la escases de vegetación, en los sitios intervenidos ocurre lo contrario. Por tal razón los ganaderos suelen ingresar sus animales a pastar donde fueron construidas las obras, generando un daño que puede ser irreversible debido a que estas al inicio son muy vulnerables.

En el sentido de lo anterior, con el afloramiento de la vegetación aparecen casi que de inmediato los animales nativos aéreos y terrestres, que en la actualidad son categorizadas como amenazadas debido a la perturbación de su hábitat, la caza indiscriminada, entre otras cosas. Con la presencia en el sitio de los 
mencionados animales, principalmente los terrestres, el hombre suele realizar capturas con los mismos métodos que los hicieron desaparecer de la zona, entre lo que es destacado las quemas de la vegetación.

De acuerdo a lo anterior, para garantizar la sostenibilidad de las obras de bioingeniería es indispensable realizar una fuerte campaña de educación ambiental al momento de construirlas. Esa pedagogía debe trascender durante un tiempo posterior a la construcción. En complemento las autoridades competentes deben implementar medidas sociales coercitivas dentro de la legalidad, contra las personas que atenten contra dichos patrimonios ambientales.

\section{Agradecimientos.}

A la Secretaria de Agricultura, Ganadería y Medio Ambiente del Municipio de Sincelejo y la Fundación Autónoma para el Desarrollo Colombiano (FUNADEC).

\section{ReFERENCIAS}

1. Suarez J. Control de erosión en zonas tropicales. Bucaramanga, Colombia; División Editorial y de Publicaciones Universidad Industrial de Santander; 2001. URL Disponible en: http://www.erosion.com.co/presentaciones/category/9-control-de-erosion-en-zonastropicales.html?download $=66: 775$-controldeerosionenzonastropicales-completo

2. Cevallos D, Toro L. Evaluación de la susceptibilidad a la erosión por el cambio de cobertura debido a la minería, en el Municipio de Anorí, Antioquia, Colombia. Revista Gestión y Ambiente. 2012; 15(3):51-64. URL Disponible en: https://revistas.unal.edu.co/index.php/gestion/article/view/36280

3. Schosinsky G. Cálculo de la recarga potencial de acuíferos mediante un balance hídrico de suelos. Rev Geol Amér. 2006; 34(35):13-30. DOI: https://doi.org/10.15517/rgac.v0i34-35.4223

4. Rivera J, Lal R, Amézquita E, Mesa O, Chaves B. Movimiento del agua y procesos fundamentales en la erosión de cinco suelos de la zona cafetera central colombiana. Cenicafé.1998; 49(4):308-324.

5. Mora M, Ríos L, Ríos L. Almario J. Impacto de la actividad ganadera sobre el suelo en Colombia. Ingeniería y Región. 2017; 17:1-12. DOI: https://doi.org/10.25054/22161325.1212

6. Vergara V, Gutiérrez G, Flórez H. Evaluación de la vulnerabilidad del acuífero Morroa a contaminación por plaguicidas aplicando la metodología DRASTIC. Ingeniería \& Desarrollo. 2009; 11(26):51-64. URL Disponible en: http://rcientificas.uninorte.edu.co/index.php/ingenieria/article/viewArticle/740/4497

7. Vergara V. Evaluación ambiental del acuífero formación Morroa por aplicación de agroquímicos en su área de recarga, Sucre- Colombia. [Tesis M.Sc]. Cartagena, Colombia: Universidad de Cartagena; 2009.

8. Yaguache L, Rodrígues V, Blanco L, Hurtado, J. Análisis de la erosión laminar en cuatro tipos de cobertura vegetal, con ayuda de la RUSLE (Revised Universal Soil Loss Equation) en la microcuenca del rio Pimenta. Revista GEOESPACIAL. 2016; 13(1):30-39. URL Disponible en: http://geoespacial.espe.edu.ec/wp-content/ uploads/2017/01/Geoespacial13.1.pdf

9. Morales M. Estimación de la erosión laminar y sedimentación con modelos hidrológicos para la selección de prácticas de conservación de suelos y cambios de cobertura en la Unidad Hidrográfica de Aquin/ Saint-Louis Du Sud- Haití. [Tesis Ph.D]. Turrialba, Costa Rica. Centro Agronómico Tropical de Investigación y Enseñanza; 2014. URL Disponible en: http://repositorio.bibliotecaorton.catie.ac.cr/bitstream/ handle/11554/7082/Estimacion_de_la_erosion_laminar.pdf?sequence $=1$

10. Osterkamp W. Annotated Definitions of Selected Geomorphic Terms and Related Terms of Hydrology, Sedimentology, Soil Science and Ecology. Virginia: Geological Survey; 2008. URL Available in: https:// pubs.er.usgs.gov/publication/ofr20081217

11. Nyssen J, Poesen J, Moeyersons J, Luyten E, Veyret-picot M, Deckers J, et al. Impact of road building on gully erosion risk: A case study from the Northern Ethiopian Highlands. Earth Surface Processes and Landforms. 2002; 27(12):1267-1283. DOI: https://doi.org/10.1002/esp.404

12. Ramos A, Trujillo-vela M, Prada L. Análisis descriptivos de procesos de remoción en masa en Bogotá. Obras y Proyectos. 2015; (18):63-75. DOI: http://dx.doi.org/10.4067/S0718-28132015000200006 
13. Barrientos Z. Problemática de los ciclos biogeoquímicos, hidrológico y de nutrientes en la meseta central de Costa Rica. Revista Posgrado y Sociedad. 2010; 10(1):23-37. DOI: http://dx.doi.org/10.22458/rpys.v10i1.1873

14. Alcaldía de Morroa. Plan de Desarrollo Estratégica Morroa Sucre 2008 - 2011. 2008. URL Disponible en: http://cdim.esap.edu.co/bancomedios/Documentos\%20PDF/morroa\%20-\%20sucre\%20pd \%20estrategica\%202008-20011.pdf

15. Cardozo J. El matarraton (Gliricidia sepium) en la alimentación de rumiantes. [Tesis Esp]. Bogotá: Universidad Nacional Abierta y a Distancia; 2013. URL Disponible en: https://stadium.unad.edu.co/preview/UNAD.php? url=/bitstream/10596/1076/1/93117211.pdf

16. Julca A, Meneses L, Blas R, Bello, S. La materia orgánica, importancia y experiencias de su uso en la agricultura. IDESIA. 2006; 24(1):49-61. URL Disponible en: https://www.uv.mx/personal/tcarmona/files/2010/08/ Julca-et-al-2006.pdf

17. Flórez G. Efectividad de la bioingeniería para el tratamiento de la erosión y los movimientos en masa en laderas. [Tesis M.Sc]. Manizales, Colombia: Universidad de Manizales; 2014. URL Disponible en: http://ridum.umanizales.edu.co:8080/xmlui/bitstream/handle/6789/1282/ Florez_Florez_Gloria_del\%20Socorro_2014.pdf?sequence $=1$

18. De la Ossa J, Pérez A, Montes D. El programa de Zootecnia: retos de investigación e innovación para Colombia. Rev Colombiana Cienc Ani - RECIA. 2018; 10(2):109-110. https://doi.org/10.24188/recia.v10.n2.2018.647

19. Cuervo A, Narváez W, Hahn Von CH. Características forrajeras de la especie Gliricidia sepium (Jacq.) Stend, fabace. Bol Cient Mus Hist Nat. 2013; 17(1):33-45. URL Disponible en: http://www.scielo.org.co/pdf/bccm/ v17n1/v17n1a03.pdf

Los autores permiten a RECIA reimprimir el material publicado en él. En caso de que un autor quiera traducir o usar una publicación parcial o completa de nuestro Diario, el autor debe obtener un permiso por escrito del editor de la revista.

CC BY 